\title{
Evaluation of Laboratory Request and Report Form Completeness and Legibility in Tuberculosis Diagnostic Laboratory at Hiwot Fana Specialized University Hospital, Harar, Ethiopia
}

\author{
Dagne Bodena ${ }^{1, *}$, Mekuria Edae ${ }^{1}$ and Zerihun Ataro ${ }^{2}$ \\ ${ }^{1}$ Hiwot Fana Specialized University Hospital, College of Health and Medical Sciences, Haramaya University, Ethiopia \\ ${ }^{2}$ Department of Medical Laboratory Sciences, College of Health and Medical Sciences, Haramaya University, Ethiopia
}

*Corresponding author: Bodena D, Hiwot Fana Specialized University Hospital, College of Health and Medical Sciences, Haramaya University, PO Box: 235, Harar, Ethiopia, Tel: +251910127241; E-mail: dagne2004bodena@gmail.com

Received: 04 Apr, 2020 | Accepted: 22 Apr, 2020 | Published: 28 Apr, 2020

Citation: Bodena D, Edae M, Ataro Z (2020) Evaluation of Laboratory Request and Report Form Completeness and Legibility in Tuberculosis Diagnostic Laboratory at Hiwot Fana Specialized University Hospital, Harar, Ethiopia. J Clin Lab Med 5(1): dx.doi.org/10.16966/2572-9578.134

Copyright: (C) 2020 Bodena D, et al. This is an open-access article distributed under the terms of the Creative Commons Attribution License, which permits unrestricted use, distribution, and reproduction in any medium, provided the original author and source are credited.

\section{Abstract}

Background: The laboratory plays a crucial role in patient care. Laboratory requests and reports forms are used as a communication agent between clinicians and laboratory professionals. Completing laboratory request and report forms reduce errors associated with customers' results that have negative impact on both diagnosis and management of the patients including extra loss of resource and time.

Objective: This study aimed to Evaluation of Laboratory Request and Report Form Completeness and legibility in Tuberculosis Diagnostic Laboratory at Hiwot Fana Specialized University Hospital, Harar, Ethiopia from September 1, 2019 to December 30, 2019.

Methods: A cross-sectional study was conducted on completeness and legibility of 1,356 patient's laboratory request and report. The data were collected on excel spread sheet and entered into EpiData software 4.2 and analyzed by using the Statistical Product and Service Solution version 21.

Results: From a total of 1,356 laboratory request and report evaluated, $72.9 \%$ of laboratory requests and $81.7 \%$ of laboratory reports were fully completed with all information. The only $100 \%$ complete information appeared on both laboratory request and report form was the patient name. About $74 \%$ of laboratory request and $78 \%$ of laboratory report are concise and legible handwriting.

Conclusion: According to this study about $27 \%$ of the information on both laboratories request and $18 \%$ reports form has not well completed with all parameters, and around $26 \%$ of clinician and $24 \%$ of laboratory personnel handwriting were not legible that faces customers for unnecessary wastage of time and resources. Furthermore, it has a great role for lengthening turnaround time of result dispatch. Therefore, to fill this gap all important information concerning all parameters in both request and report formats must be clearly addressed with legible hand witting.

Keywords: Completeness; Legibility; Laboratory request; Laboratory report;

List of Abbreviations: IHRERC: Institutional Health Research Ethics and Review Committee; HFSUH: Hiwot Fana Specialized University Hospital; OPD: Outpatient Department; TAT: Turn Around Time; TB: Tuberculosis; MDR TB: Multidrug Resistance of Tuberculosis; ISO: International Standardization Organization; SPSS: Statistical Product and Service Solutions

\section{Background}

Medical laboratory request and report forms are used as a link of communication among physician, laboratory staff, patients, and other health professionals [1]. Approximately $60-70 \%$ of all decisions about patients' diagnosis, hospital admission, treatment and discharge depend on medical laboratory results [2,3]. Effective completion of these forms revealed care and conscientiousness in one's responsibility and to ensure the correct communication among them.
Most of hospitals and health centers in the developing countries give little attention to the information supplied in laboratory request and report form. Incomplete lab request and report have negative impact on communication and patients' results may be wrongly used by another patient or sent to another clinician that can result in a life-threatening outcome and difficulty in control infectious disease like multidrug resistance of tuberculosis as well as cause unnecessary wastage of time and resources. Particularly if a critical result is there 
in the laboratory report, a patient is in imminent danger unless appropriate therapy is initiated promptly [4-6].

International Organization for Standardization (ISO) 15189 states that the request and report form shall contain patients' a unique identifiers, like gender, age, contact address, name, card number, name and signature of test requester, type of sample, date and time of primary sample collection; date and time of sample receipt, a signature of the laboratory personnel who does the test, result approval name and signature, report date and report time [4,7-9]. So that, filling both laboratories request and report with legible and adequate manner insight a vital role in the definitive diagnosis. It also strengthens the quality management system and quality of laboratory results that ultimately have a positive outcome on patients' management and significantly increase customer satisfaction by decreasing Turn Around Time (TAT) of each result $[10,11]$.

Assessments of laboratory requests and report formats have been done in different countries. A study conducted in Nigeria revealed that information on clinical details and patient's age were only provided in $55.6 \%$, and $42.1 \%$ respectively and the least provided information was time with $0.7 \%$ and date of specimen collection with 3.6\% [10]. Another study done in Addis Ababa, Ethiopia showed that the percentage of requests with patients' card number, name, age, sex, OPD, patient history, and type of test were $77.37 \%, 100 \%, 76.67 \%$, $95.19 \%, 80.2 \%, 76.9 \%$, and $97 \%$ respectively [4].

Additionally, inappropriate filling of these laboratory forms come up with untrusted customer on the organizational service which evidenced by study done at eastern Ethiopia hospitals in which customer satisfied by the laboratory services providers was $80.0 \%$ [12] and another study done at Debre Markos hospital that level of patient satisfaction towards clinical laboratory services was only $48.3 \%$ that likelihood high in patient with who had an occasion of missing results [13].

Generally, adequate and legible filling of both laboratory request and report has a great benefit that increases the flow of information between clinician and laboratory service provider to give a better diagnosis as per of international standard quality in the health system as well as increase level of customer satisfaction towards clinical laboratory services. In Ethiopian, most of health care services are not computerized system rather than it is communicated with manual system like requesting and reporting result with hand writing, which results out illegibility that brings missing of patients result. Lack of enough training and low understanding on good clinical laboratory practice challenge to miss essential parameter in request/report forms if it is not evaluated appropriately before sample reception by laboratory professionals or vice versa by physicians on request paper [4]. However, there is limited research reported from Ethiopia. Therefore, this study aimed to evaluate the completeness and legibility of information on Tuberculosis (TB) laboratory request and report forms at Hiwot Fana Specialized University Hospital, Harar, Ethiopia.

\section{Materials and Methods}

\section{Study area and period}

Harar is the capital city of Harari regional state and one of the oldest and most historical cities of Ethiopia which is $526 \mathrm{Km}$ away from Addis Ababa the capital city of Ethiopia. According to the central statistical agency of 2007, the total population of Harari Regional state was 183,415 , of whom 91,099 were females and 92,316 were males. This study was conducted on Tuberculosis laboratory diagnosis at Hiwot Fana Specialized University Hospital (HFSUH), Harar, Ethiopia in the period of September 1, 2019 to December 30, 2019. Hiwot Fana Specialized University Hospital is one of the biggest hospitals in Eastern Ethiopia that currently serves about 5.6 million people around Harari regional state and neighboring regions like Dire Dawa administrative council, Oromiya, and Ethiopian Somali Regional State. The laboratory services provided by this hospital include: parasitology tests, clinical chemistry tests, urine analysis, serology, hematology, CD4 count, Genexpert and others [14].

\section{Study design and sampling technique}

A cross-sectional study was conducted by following convenient selection method of laboratory request and report form concerning to the completeness and legibility that sent from Tuberculosis (TB) clinic and different department of Hiwot Fana Specialized University Hospital (HFSUH) and from other referral center.

\section{Method of data collection and study instrument}

Standardized checklist was developed from International Standardization Organization (ISO) 15189, and a laboratory quality management for clinical laboratories to evaluate the request and report forms during data collection. Information on the laboratory request during entry to the laboratory Tuberculosis $(\mathrm{TB})$ reception and laboratory report result before handled by patients were collected by using an excel spreadsheet as yes or no for the presence or absence of the particular information including legibility of their hand writing. This information contain name, age, sex, card number, contact address, type of sample, name, date, and signature of clinician who order the test, name of the test examiner and signature, date and time of specimen received, date and time of result dispatched, verifier name and a signature, and report date. This laboratory requests sent to laboratory Tuberculosis (TB) diagnosis from different department and Tuberculosis (TB) clinic are not directly returned, instead, it retained and use similar laboratory report forms to dispatch. Both laboratory request and report formats used by the clinician, patients and laboratory personnel during the data collection period were included in the study and those formats that are not used for diagnosis are excluded from this study.

\section{Quality Control}

Pre-tested check list was used to data collection to increase the quality of data; in addition training was given for data collectors. The data was collected by two trained laboratory technicians. The collected data were double crosschecked by the investigator to reduce mistakes.

\section{Statistical Analysis}

Depends on our resource data the collected data were entered into EPI-data software Version 4.2 and analyzed by using SPSS (IBM Statistics, USA) software version 21. Descriptive statistics of different variables were evaluated and presented in the form of texts, and tables. Summary measures such as percentages were used to generalize the results accordingly.

\section{Results}

In this study, a total of 1,356 laboratory requests and 1,356 laboratory reports have been evaluated for the completeness and legibility status of necessary information as stated in both formats. From a total of 1,356 laboratory requests sent to the Tuberculosis (TB) laboratory department from different department and Tuberculosis (TB) clinic; the proportion of completed laboratory requests with patient name, age, sex, patient history, and address are $100 \%, 99.6 \%, 99 \%, 97.1 \%$, and $79.7 \%$ respectively. Concerning to information filled by the clinician; 
the name of the clinician ordering the test was $94.2 \%$, while $96.8 \%$ of clinicians were signed by them. The completed type of sample ordered for testing was $74.3 \%$ from the requests. About $74 \%$ of laboratory requests were concise and legible handwriting (Table 1).

The information indicated on laboratory report forms are shown in (Table 2).

From the in completed laboratory requests, 728(53.7\%) was dispatched out of the stated Turn Around Time (TAT), while 601 (95.7\%) of completed laboratory requests, have dispatched within the stated TAT of Hiwot Fana specialized University Hospital laboratory (Table 3).

\section{Discussion}

Laboratory results have a great contribution to patient care and

Table 1: Completeness of TB diagnosis laboratory request format at Hiwot Fana Specialized University Hospital, Harar, from September to December, 2019.

\begin{tabular}{|l|c|c|c|c|}
\hline \multirow{2}{*}{ Lab request parameter } & \multicolumn{2}{|c|}{ Complete } & \multicolumn{2}{c|}{ Incomplete } \\
\cline { 2 - 5 } & Number & Percent (\%) & Number & Percent (\%) \\
\hline Name of patient & 1356 & 100 & 0 & 0 \\
\hline Age & 1350 & 99.6 & 6 & 0.4 \\
\hline Sex & 1351 & 99.6 & 5 & 0.4 \\
\hline MRN & 1347 & 99.3 & 9 & 0.7 \\
\hline Address & 1081 & 79.7 & 275 & 20.3 \\
\hline History of patient & 1317 & 97.1 & 39 & 2.9 \\
\hline Type of sample test & 1008 & 74.3 & 348 & 25.7 \\
\hline $\begin{array}{l}\text { Ordered clinician name } \\
\text { and sign }\end{array}$ & 1277 & 94.2 & 79 & 5.8 \\
\hline Date of test ordered & 1273 & 93.9 & 83 & 6.1 \\
\hline $\begin{array}{l}\text { Time of sample } \\
\text { collection }\end{array}$ & 1301 & 95.9 & 55 & 4.1 \\
\hline $\begin{array}{l}\text { Legibility of clinician } \\
\text { writing on request paper }\end{array}$ & 1004 & 74 & 356 & 26 \\
\hline
\end{tabular}

Abbreviation: MRN: Medical Record Number

Table 2: Completeness of Tuberculosis (TB) diagnosis laboratory report parameter at Hiwot Fana Specialized University, Harar, from September to December, 2019.

\begin{tabular}{|l|c|c|c|c|}
\hline \multirow{2}{*}{ Lab report parameter } & \multicolumn{4}{|c|}{ Laboratory report parameter } \\
\cline { 2 - 5 } & $\begin{array}{c}\text { Complete } \\
\text { (No) }\end{array}$ & $\begin{array}{c}\text { Percent } \\
\text { (\%) }\end{array}$ & $\begin{array}{c}\text { Incomplete } \\
\text { (No) }\end{array}$ & Percent (\%) \\
\hline Name of patient & 1356 & 100 & 0 & 0 \\
\hline Age & 1301 & 95.9 & 55 & 4.1 \\
\hline Sex & 1347 & 99.3 & 9 & 0.6 \\
\hline MRN & 1355 & 99.9 & 1 & 0.08 \\
\hline $\begin{array}{l}\text { Name of lab test done } \\
\text { and sign }\end{array}$ & 1303 & 96.1 & 53 & 3.9 \\
\hline Report date & 1356 & 100 & 0 & 0 \\
\hline $\begin{array}{l}\text { Name of test approval } \\
\text { and sign }\end{array}$ & 1108 & 81.7 & 248 & 18.3 \\
\hline Report time & 1342 & 99 & 14 & 1 \\
\hline $\begin{array}{l}\text { Legibility of lab } \\
\text { personnel writing on } \\
\text { report form }\end{array}$ & 1058 & 78 & 298 & 22 \\
\hline
\end{tabular}

Table 3: Relationship between completion of laboratory requests form and TAT at Hiwot Fana Specialized University Hospital, Harar, from September to December, 2019.

\begin{tabular}{|c|c|c|c|c|c|}
\hline \multicolumn{2}{|c|}{ Complete Laboratory Requests } & \multicolumn{3}{|c|}{ Incomplete Laboratory Request } \\
\hline $\begin{array}{c}\text { No of } \\
\text { lab } \\
\text { request }\end{array}$ & $\begin{array}{c}\text { Test } \\
\text { dispatched } \\
\text { within TAT }\end{array}$ & $\begin{array}{c}\text { Test } \\
\text { dispatched } \\
\text { out of the } \\
\text { stated TAT }\end{array}$ & $\begin{array}{c}\text { No of } \\
\text { lab } \\
\text { request }\end{array}$ & $\begin{array}{c}\text { Test } \\
\text { dispatched } \\
\text { within TAT }\end{array}$ & $\begin{array}{c}\text { Test } \\
\text { dispatched out } \\
\text { of the stated } \\
\text { TAT }\end{array}$ \\
\hline 628 & $\begin{array}{c}601 \\
(95.7 \%)\end{array}$ & $27(4.3 \%)$ & 728 & $\begin{array}{c}600 \\
(82.4 \%)\end{array}$ & $128(17.7 \%)$ \\
\hline
\end{tabular}

estimated to affect about $70 \%$ of medical diagnoses [2]. The correct interpretation of the test results is a core point to patient management that highly based on the information given on the request and report form. Several studies have shown deficiencies in the filling of laboratory requests and report forms world widely [5,11,15-18].

In this study, the overall fully completed request and report format is only $72.9 \%$ and $81.7 \%$ respectively. This is higher than comparing proportionally a study from Nigeria in which only $5(0.2 \%)$ was fully completed [11]. However, lower than study from Addis Ababa, Ethiopia that $77.37 \%$ of lab requests and $87.15 \%$ of laboratory report formats were fully completed [4]. The observed difference might be due to different level of laboratory quality management implementation status/may some laboratories undergo accreditation process or others may not.

The name of the patient in this study was the only parameter that presents in all the laboratory requests and reports forms. This is proportion is similar to the findings of other studies $[15,17,19-21]$ that the name of the patient was fully completed. This might be due to the fact that patients' names were as simple as it appears to be first in many requests and report format [6] and may also be due to rejected at the reception of any request form that has no patients' name.

The age was appeared in about $99.6 \%$ in request laboratory formats and $95.9 \%$ in a report format in this study. This proportion is higher than the finding of $42.1 \%$ and $39 \%$ in Nigeria [11,15] and similar to study from North India that age was completed with $99.8 \%$ of Blood bank and $99.7 \%$ blood chemistry laboratory requests [16]. The observed difference might be due to a different level of awareness of clinician and laboratory personnel about the importance of demographic and clinical information of patient that may have a negative influence on many diseases conditions and drug dose for treatment which specifically depend on certain age groups.

In this study, sex was completed in $99 \%$ of both laboratory requests and report forms. This proportion is more comparable with studies reported from Tanzania 99.5\% [6] and 97.8\% in Nigeria [11]. So that sex is an important parameter that clinicians use appropriately during interprets test results. The hospital registration number provided was about $99 \%$ in both requests and report lab in this study. This is higher than the results of $78.8 \%$ and $87 \%$ from Nigeria $[11,15]$. However, it is more comparable with a study from North India with 97\% [17] and $100 \%$ Kenya [20]. The hospital registration number is important when samples from different subjects have similar names and other information in identifying and sorting out both the subject and samples as to fast-tracking the process of making the diagnosis.

The clinical history of patients' appeared in this study was about $97.1 \%$. This proportion is higher than study from Nigeria $75 \%, 84.5 \%$ 
$[15,19]$ and Kenya $14.9 \%$ [20]. However, it was comparable with a report from North India 98\% [17]. The difference might be due to different level of laboratory quality management implementation status/may some laboratories undergo accreditation process or others may not. Filling clinical history appropriately is important for correct interpretation of laboratory results and omitting of clinical information leads to unnecessary additional tests, resource loss and other demand implications.

About $79.7 \%$ of patients' address was provided in this study. This proportion is lower than report from Nigeria 90.4\% [6] and $96.9 \%$ [19]. The occurred difference might be due to different level of knowledge about the importance of patients' address. This may help in an epidemiological survey of a particular disease observed to be common in a certain locality and especially in cases of critical results like Multidrug Resistance of Tuberculosis (MDR-TB) which require urgent action to control the infectiousness of the disease. Further, it has great implication to reduce the disease by giving care and testing in pre-movement.

The names of physicians and signature and date of test ordered appeared in $94 \%$ and $93.8 \%$ respectively. This proportion is consistent with a report of $93.2 \%$ and $93.8 \%$ in Nigeria [11,21]. However, it was higher than the study from Tanzania $7.7 \%$ [20]. This may be due to variations to considering time constraints that might be experienced in a busy clinic and accident department.

Name and signature of person testing the sample, name and signature person approved the result were provided in $96.1 \%$ and $72.9 \%$ of reports, respectively. This proportion is higher than the study from Addis Ababa, Ethiopia in which name of test done and a sign was $79.2 \%$ and test done approval name and sign was $15.6 \%$. The gap of test done approval name and sign was a great impact on result reliance by customer and rechecking of error before the result was dispatched. From the assessed request forms, 99\% of laboratory report has the time of result released. This proportion is higher compared to the study from Addis Ababa, Ethiopia that only 55.6\% has the time of result released. The observed difference might be due to various use of information system that some Laboratory Applies Information System (LIS) or computerized system and others may did not use it.

About $26 \%$ of laboratory request and $22 \%$ of laboratory report are illegible handwriting that faces challenge in most health care services. Most of lab request and report in Ethiopia are not computerized that legibility of one's lab request and report are dependable on individual handwriting. So, an illegible handwriting weaken the communication between clinician and laboratory professionals during requesting and reporting result exposes patients to extra wastage of money and time, due to losing of results.

From the total of 728 incomplete laboratory requests, 600(82.4\%) were done within stated Turn Around Time (TAT) and the rest of 128 (17.7\%) was reported in the stated Turn Around Time (TAT). Moreover, out of 628 completed laboratory requests, 601 (95.7\%) have reported within stated Turn Around Time (TAT) and the rest 27 (4.3\%) have taken a longer time than stated Turn Around Time (TAT), that implies incomplete laboratory requests have taken more time than that of the completed ones. During the study period about 24 samples were rejected by laboratory staffs. Actually this problem is somewhat not only lack of incompleteness of laboratory requests; but also unlabeled sample, and especially those problems that can directly influence the test results of the patients or specimens with unknown essential patient information were rejected. If we were rejecting all samples with incomplete forms, this would mean at least $27 \%$ of the request forms were rejected which leads to challenging patients in case of resource and time wastage.

\section{Conclusion}

The study revealed that there were incomplete in filling of the required information in both the laboratory request and report form. Fully completing both forms have advantage in the identification of the gap occurs and the responsible personnel for each work in the testing of sample. Additionally, these completed formats have great benefit in simplifying communication between health professionals and patients. Furthermore, completed formats reduces the workload, ensures quality and timely results to the specified patient.

\section{Recommendation}

Based on this study, all Clinicians and laboratory professionals must be properly fill all laboratories request and report parameter forms and also being part of their daily activities. Upgrade the awareness of clinicians and laboratory personnel on the importance of adequate and complete filling of laboratory request and result forms. Furthermore, regular follow up should be provider to all laboratory personnel and clinician. Other study needed to identify the reasons for the inadequate filling of the laboratory request forms by the clinicians and result form by laboratory personnel should be carried out.

\section{Acknowledgments}

We would like to express our great gratitude to Hiwot Fana specialized university Hospital for permission to conduct the study and also individuals that supports us in conducting this study.

\section{Author's Note}

D.B conceived and designed the study; participated in data collection, analysis, interpretation and write-up; drafted the manuscript and critically revised the manuscript. M.E designed the study; participated in data collection, analysis, interpretation and write-up; drafted the manuscript and critically revised the manuscript. Z.A designed the study; participated in data collection, analysis, interpretation and write-up; drafted the manuscript and critically revised the manuscript. All authors made substantial intellectual contributions to data analysis and gave final approval of the version to be published and agree to be accountable for all aspects of the work.

\section{Ethical Consideration}

The study was approved by the ethics of the College of Health and Medical Sciences, Haramaya University the Institutional Research Ethics Review Committee. Permission was obtained from Hiwot Fana Specialized University Hospital, where the study was conducted. Based on the objective of the study, an official letter was sent to Hiwot Fana Specialized University Hospital. Confidentiality related to patient, laboratory personnel and physician were kept/not captured on the datasheet for analysis.

\section{Declaration of Conflict Interests}

The authors declare that they have no competing interests in this work and with publication of this article.

Funding-No fund was received to conduct this study.

\section{References}

1. Opperman CJ (2018) Completing laboratory request forms diligentlywhen did it become optional? S Afr Med J 108: 12341. 
2. Mayo Clinic School of Health Science (2016) Medical Laboratory Sciences.

3. Plebani $\mathrm{M}$ (2006) Errors in clinical laboratories or errors in laboratory medicine ? Clin Chem Lab Med 44: 750-759.

4. Tewabe H, Haile B, Siyum B, Mihret G, Dagne W (2019) Evaluation of Lab Request and Report Formats Completeness and its Advantage for both Health Professionals and Patients in a Health Center, Addis Ababa, Ethiopia. J Clin Lab Med 4.

5. Nutt L, Zemlin EA, Erasmus TR (2008) Incomplete laboratory request forms: the extent and impact on critical results at a tertiary hospital in South Africa. Ann Clin Biochem 45: 463-466.

6. Alagoa PJ, Udoye EP (2015) Laboratory Request Forms- How Well do Doctors Fill Them? A Look at the Practice at the Niger Delta University Teaching Hospital, Okolobiri, Bayelsa State, Nigeria. Nigerian Health J 15: 14-17.

7. ISO (2012) Medical laboratories-Requirements for quality and competence. $3^{\text {rd }}$ Edition, International Standard 15189.

8. Horton TC, NDO Lab, SVCS P (2014) Valid Laboratory Orders for Outpatient. Policy.

9. Ministry of Health, Ethiopia Public Health Institute (2014) Implementation Guideline for GeneXpert MTB/RIF Assay in Ethiopia. Federal Democratic Republic of Ethiopia, Addis Ababa, Ethiopia.

10. Olumide BO, Joel AO, Paul ME, Angela CN, Steve OK, et al. (2019) Assessment of Patients' Medical Laboratory Request Forms for Compliance in Jos University Teaching Hospital, Jos-Nigeria. Am J Biomed Sci Res 6

11. Oyelekan AA, Ojo OT, Olawale OO, Adeleye OO, Sogebi OA, et al. (2018) Pattern of completion of Laboratory Request Forms in a tertiary health facility. Ann of Health Research 4: 155-161.

12. Teklemariam Z, Mekonnen A, Kedir, Kabew G (2013) Clients and clinician satisfaction with laboratory services at selected government hospitals in eastern Ethiopia. BMC Res Notes 6: 15.
13. Alelign A, Belay AY (2019) Patient satisfaction with clinical laboratory services and associated factors among adult patients attending outpatient departments at Debre Markos referral hospital, Northwest Ethiopia. BMC Res Notes 12: 517.

14. Hiwot Fana specialized University hospital annual report (2019).

15. Adamu S, Mohammed, A El-Bashir MJ, Abubakar DJ, Mshelia SD (2018) Incomplete Patient Data on Chemical Pathology Laboratory Forms in a Tertiary Hospital in Nigeria. Ann Trop Pathol 9: 47-49.

16. Dogethera MA, Muallem YA, Househ $M$, Saddik B, Khalifa M (2016) The impact of automating laboratory request forms on the quality of healthcare services. J Infect Public Health 9: 749756

17. Kansay S, Verma M (2016) Evaluation of request forms received in blood bank and biochemistry laboratory in a teaching hospital in North India: A comparative study of preanalytical errors. J Nat Accred Board Hosp Healthcare Providers 3: 20-26.

18. Taichman DB, Backus J, Baethge C, Bauchner H, de Leeuw PW, et al. (2016) Sharing Clinical Trial Data: A Proposal from the International Committee of Medical Journal Editors. Rev Med Chil 144: 11-13.

19. Kipkulei JC, Lotodo TC (2019) Evaluation of the Completeness in the Filling of Laboratory Request Forms Submitted to the Haematology Laboratory at a Tertiary Hospital in Kenya. Health 11: 862-868.

20. Makubi An, Meda C, Magesa A, Minja P, Mlalasi J, et al. (2012) Audit of clinical-laboratory practices in haematology and blood transfusion at Muhimbili National Hospital in Tanzania. Tanz J of Heal Res 14: 257-262.

21. Adegoke OA, Idowu AA, Jeje OA (2011) Incomplete laboratory request forms as a contributory factor to preanalytical errors in a Nigerian teaching hospital. African J of Bioche Res 5: 82-85. 